\title{
Primer Registro de Tinamú Serrano Nothocercus bonapartei (Tinamiformes: Tinamidae) en la cordillera del Cóndor, Sureste de Ecuador
}

\author{
Leonardo Ordóñez-Delgado*, Fausto López, Fabián Reyes-Bueno \\ Departamento de Ciencias Biológicas, Universidad Técnica Particular de Loja. CP: 11-01-608. Loja, Ecuador. \\ *Autor para correspondencia/Corresponding author, e-mail: lyordonez2@utpl.edu.ec \\ Editado por/Edited by: Esteban Guevara. \\ Recibido/Received: 06-07-2016. Aceptado/Accepted: 29-03-2017. \\ Publicado en línea/Published on Web: 18-04-2017. \\ DOI: \\ First Record of Highland Tinamou Nothocercus bonapartei (Tinamiformes: Tinamidae) \\ in the cordillera del Cóndor, Southeast Ecuador
}

\begin{abstract}
Resumen
El Tinamú Serrano Nothocercus bonapartei es un ave rara, de comportamiento sigiloso y poco conocida que habita bosques densos y poco disturbados al este de los Andes en Ecuador, donde existen pocos registros. Presentamos los primeros registros de la presencia de esta especie en la cordillera del Cóndor, un macizo montañoso aislado de los Andes orientales.
\end{abstract}

Palabras Clave. Aves, Nueva Distribución, Zamora Chinchipe.

\begin{abstract}
The Highland Tinamou Nothocercus bonapartei is a rare, secretive, and poorly known bird that inhabits dense and undisturbed forests in the eastern Andes of Ecuador, where it is unfrequently recorded. We present the first records of this species in the cordillera del Cóndor, a mountain massif isolated from eastern Andes.
\end{abstract}

Keywords. Birds, New Distribution, Zamora Chinchipe.

La cordillera del Cóndor es un sistema montañoso aislado de la cordillera de los Andes, ubicado en el sureste de Ecuador y noreste de Perú (Freile \& Santander, 2005). Se conecta con los Andes solamente por un pequeño ramal montañoso ubicado en la parte sur de la cuenca hidrográfica del Nangaritza. La cordillera del Cóndor posee una longitud de aproximadamente $150 \mathrm{~km}$ y una elevación máxima de $2900 \mathrm{~m}$ en el sector de Cerro Plateado (Neill, 2005). Esta cordillera posee extraordinarios niveles de endemismo, principalmente debido a su aislamiento y la existencia de varios ecosistemas que se desarrollan sobre algunas mesetas de arenisca (Schulenberg \& Awbrey, 1997; Neill, 2005; Jadán \& Aguirre, 2011). Sin embargo, la cordillera del Cóndor se considera una de las áreas menos exploradas de Ecuador (Ridgely \& Greenfield, 2001; Montalvo, 2012; Freile et al., 2014). Varios esfuerzos de investigación ornitológica en los últimos años han permitido lograr una mejor documentación de la distribución, historia natural y ecología de las especies de aves en la zona: Ágreda et al., (2005), Loaiza et al., (2005), Krabbe \& Ahlman (2009), Freile et al., 
(2011a, b, 2014), Montalvo (2012). En la cordillera del Cóndor hay evidencia de la presencia significativa de especies de aves de los Andes y la Amazonía (Krabbe \& Sornoza, 1994; Balchin \& Toyne, 1998; Freile et al., 2014). Hasta la actualidad se han registrado 535 especies de aves en la porción ecuatoriana de la cordillera del Cóndor, 14 de estas especies se consideran globalmente amenazadas y algunas poseen una distribución restringida a hábitats específicos de la misma (Freile et al., 2011a, b, 2014). Todos estos elementos han contribuido a que la cordillera del Cóndor sea reconocida en Ecuador como un Área de Importancia para la Conservación de Aves (Freile \& Santander, 2005).

El Tinamú Serrano Nothocercus bonapartei es una de las dos especies del género que están presentes en Ecuador (Ridgely \& Greenfield, 2001; Cabot et al., 2014). Se distribuye desde Costa Rica y Panamá, pasando hacia el sur por Venezuela, Colombia, Ecuador y Perú (Cabot et al., 2014; BirdLife International, 2015; Gomes \& Kirwan, 2015). En Ecuador, su distribución comprende los bosques montanos de las estribaciones orientales de los Andes entre 1600 y 2200 m de altitud (Fjeldså \& Krabbe, 1990; Ridgely \& Greenfield, 2001, 2006; McMullan \& Navarrete, 2013), con un registro inusual logrado a $3075 \mathrm{~m}$ en Oyacachi, en la región andina nororiental del país (Krabbe et al., 1997); y, un solo registro previo documentado fuera de los Andes de Ecuador, en la cordillera de Kutukú a 1700 m (cordillera ubicada al norte de la cordillera del Cóndor, de la cual está separada por el río Santiago; Robbins et al., 1987). Debido a su amplia distribución, la Unión Internacional para la Conservación de la Naturaleza UICN clasifica N. bonapartei en la categoría de Preocupación Menor (LC, por sus siglas en inglés), a pesar de que sus poblaciones se encuentran disminuyendo, principalmente por actividades de cacería insostenible (BirdLife International, 2015). El estado de conservación de esta especie no ha sido evaluado en Ecuador. Se considera una especie poco común, solitaria y encontrada únicamente al interior de bosques con cobertura vegetal densa y poco disturbada (Gomes \& Kirwan, 2015). En este tipo de hábitats su registro visual es improbable; por ende, es más fácil oír su característica e insistente vocalización que se escucha como un repetitivo co-á, co-á, co-á, co-á..., la cual puede durar desde unos 30 segundos a varios minutos (Ridgely \& Greenfield, 2006; McMullan \& Navarrete, 2013). En la presente publicación presentamos los primeros registros del Tinamú Serrano Nothocercus bonapartei en la cordillera del Cóndor, ampliando así su distribución hacia una región del país en la cual previamente no había sido documentada.

El 1 de noviembre de 2012 durante el desarrollo de una evaluación ecológica rápida logramos grabar la vocalización de Nothocercus bonapartei en un bosque denso maduro. Este registro fue realizado en la parte baja de una meseta de arenisca en el sector La Zarza $\left(3,781389^{\circ} \mathrm{S}, 78,494722^{\circ} \mathrm{O}, 1400 \mathrm{~m}\right)$, al oeste de la cordillera del Cóndor. Las vocalizaciones se registraron de manera repetida en la misma zona durante tres días y por períodos prolongados de tiempo, sobre todo entre las $07 \mathrm{~h} 00$ y $09 \mathrm{~h} 00$, y luego de las $16 \mathrm{~h} 30$.

El 8 de noviembre de 2012, logramos un segundo registro a aproximadamente 2,5 $\mathrm{km}$ al norte del primero, en el sector El Colibrí $\left(3,761667^{\circ} \mathrm{S}, 78,499167^{\circ} \mathrm{O}, 1450 \mathrm{~m}\right)$, un bosque denso poco disturbado, relativamente plano y poco drenado, con algunas zonas anegadas. En esta ocasión, el tinamú se registró por su vocalización característica pero también logramos fotografiarlo (Figura 1). En ambos casos, la identificación de la especie se basó en las fotografías, las notas de campo y las grabaciones de audio. Las grabaciones se encuentran disponibles en la base de datos en línea xeno-canto (Ordóñez-Delgado, 2012a, b, c, d). Estas grabaciones fueron comparadas con archivos de audio confirmados de Nothocercus bonapartei (Krabbe \& Nilsson, 2003; Lysinger et al., 2005; Moore et al., 2013).

El tinamú observado en el sector El Colibrí se encontraba acompañado por una cría, por lo cual se sugiere que era un macho (en este grupo de aves son los machos quienes realizan el cuidado parental de las crías; Handford \& Mares, 1985; Ridgely \& Greenfield, 2001; Brennan, 2009; Freile, 2009; Gomes \& Kirwan, 2015). La cría siempre se mantuvo junto al adulto, y mientras este vocalizaba, la cría emitió un llamado de contacto particular, un leve hug, hug, hug, huggg..., (Ordóñez-Delgado, 2012a: minutos 1:40 a 2:04, 2012e: minutos 00:01 a 0:16, 0:38 a 0:57 y 1:07 a 1:13). Este llamado de contacto es similar a los sonidos reportados por Slud (1964) y McKay (1980) para las crías de esta especie. La cría era de aproximadamente la mitad del tamaño del adulto, coloración café oscuro, sin patrones evidentes de coloración de otro tipo en las plumas de su cuerpo, lo que difería con el adulto, en el cual si se pudo evidenciar tonalidades de color café oscuro en sus flancos y unas tenues marcas blancas en la rabadilla, además de plumas de un marcado color herrumbroso en la garganta (Figura 1).

Estos registros se obtuvieron a altitudes que se encuentran por debajo del rango altitudinal previamente conocido para la especie en Ecuador y Perú (1600 y 2200 m; Ridgely \& Greenfield, 2001, 2006; Schulenberg et al., 2007; Gomes \& Kirwan, 2015). El área donde se realizaron los registros corresponde a la formación vegetal de Bosque Montano Bajo (Sierra et al., 1999; Jadán \& Aguirre 2011), caracterizada por estar dominada por arboles de entre 5 y 25 metros de alto. Esta vegetación es de transición y se compone de especies de flora de las tierras bajas amazónicas y andinas. Algunas de las especies de plantas más representativas de esta zona son: Clarisia racemosa, Dacryodes cupularis, Miconia punctata, Nectandra cissiflora, Weinmannia elliptica y Wettinia maynensis (Jadán \& Aguirre, 2011). 


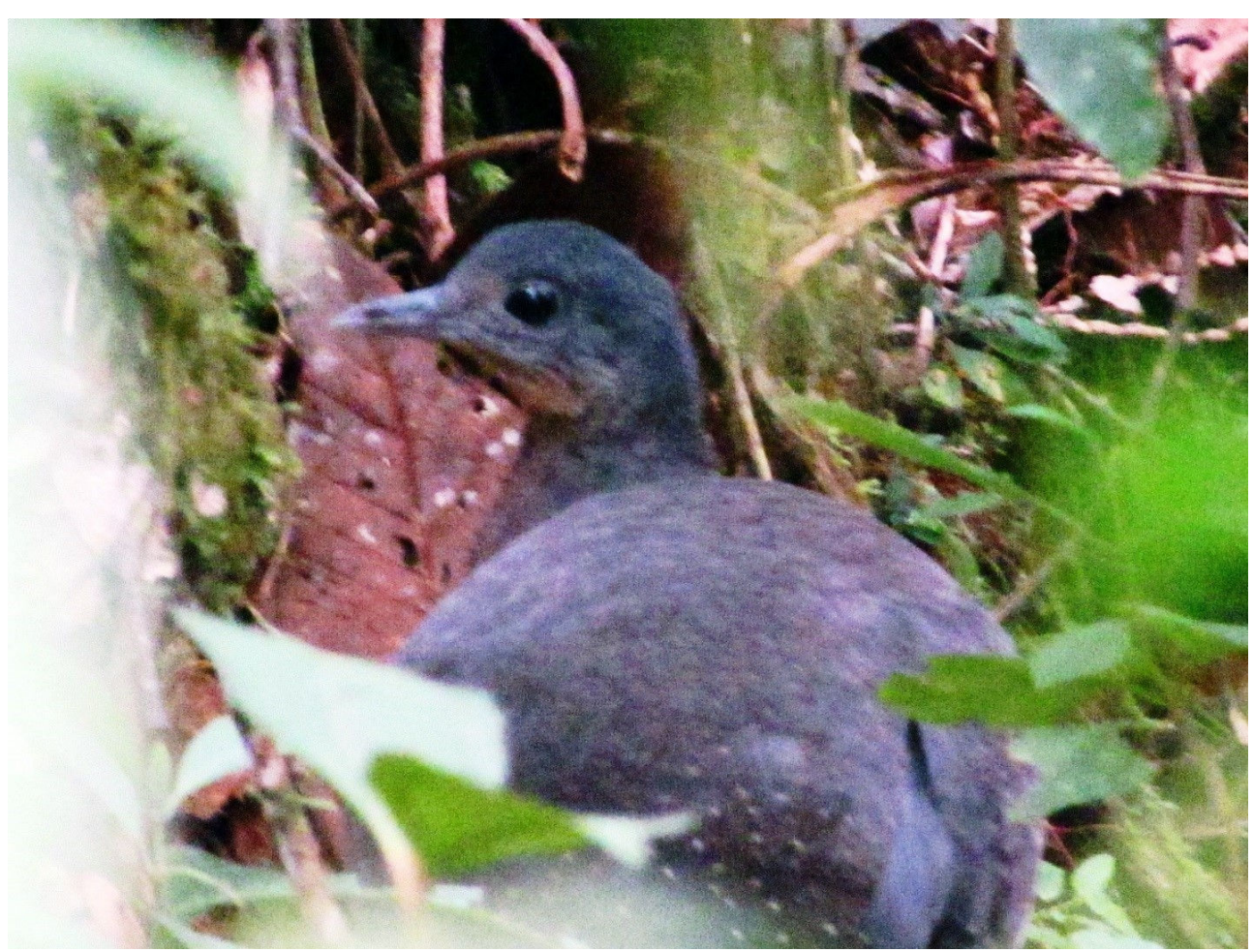

Figura 1: Adulto de Tinamú Serrano Nothocercus bonapartei fotografiado el 8 de noviembre del 2012 en el sector Colibrí - cordillera del Cóndor, sureste de Ecuador.

Estos dos registros de Nothocercus bonapartei en la cordillera del Cóndor amplían el rango de distribución de la especie en Ecuador a una zona en la cual previamente se desconocía su presencia (Figura 2). La localidad conocida más cercana corresponde a la cordillera de Numbala, a $2000 \mathrm{~m}$ en los Andes del sur del Ecuador (donde se colectó un individuo depositado en el Museo Ecuatoriano de Ciencias Naturales, MECN Or. 7231). El reporte en la cordillera de Kutukú (Robbins et al., 1987), el único fuera de los Andes, se ubica a aproximadamente $130 \mathrm{~km}$ al norte de las localidades aquí reportadas.

Estos registros permiten corroborar la afinidad de la especie por bosques poco perturbados (Gomes \& Kirwan, 2015). Es posible que su distribución en esta región sea continua en la ladera occidental de la cordillera del Cóndor, hasta encontrarse con el piedemonte andino en la parte alta de la cuenca del río Nangaritza.

El intercambio directo de individuos provenientes de los Andes es altamente improbable por la presencia del río Zamora, un accidente biogeográfico difícil de atravesar para un tinamú. Freile et al., (2014) presentaron uno de los estudios ornitológicos más completos sobre las aves de la cuenca del Nangaritza, cuyo flanco oriental es parte de la cordillera del Cóndor. En dicha publicación, se reportaron cinco especies de la familia Tinamidae: Tinamus tao, T. major, Crypturellus cinereus, $C$. soui, C obsoletus, en 11 localidades de muestreo y un rango temporal de 25 años de estudios, sin embargo estos autores no reportaron a $N$. bonapartei para la cuenca del Nangaritza, lo que podría estar ligado a la rareza de la especie.

Los tinamúes (familia Tinamidae) son el grupo de aves menos estudiado y conocido en Ecuador (Freile et al., 2006) siendo la información sobre especies particulares como $N$. bonapartei aún más escasa. Los datos aquí descritos permiten incrementar nuestro conocimiento sobre la distribución geográfica de la especie en nuestro país, así como estimaciones de su época reproductiva. Nuestro encuentro con un adulto y su cría en noviembre, concuerda con los datos publicados anteriormente en relación con la temporada de reproducción de esta especie en Colombia, en donde se ha reportado individuos con crías en noviembre y en marzo-junio (Miller, 1963; Hilty \& Brown, 1986; Cabot et al., 2014).

El registro de Nothocercus bonapartei en la cordillera del Cóndor resalta una vez más la importancia biológica y ecológica de esta región geográfica del país, la misma que enfrenta serias amenazas, principalmente por actividades de desarrollo que involucran el incremento de la deforestación para implementación de zonas agrícolas y ganaderas, además de crecientes procesos de minería legal e ilegal. 


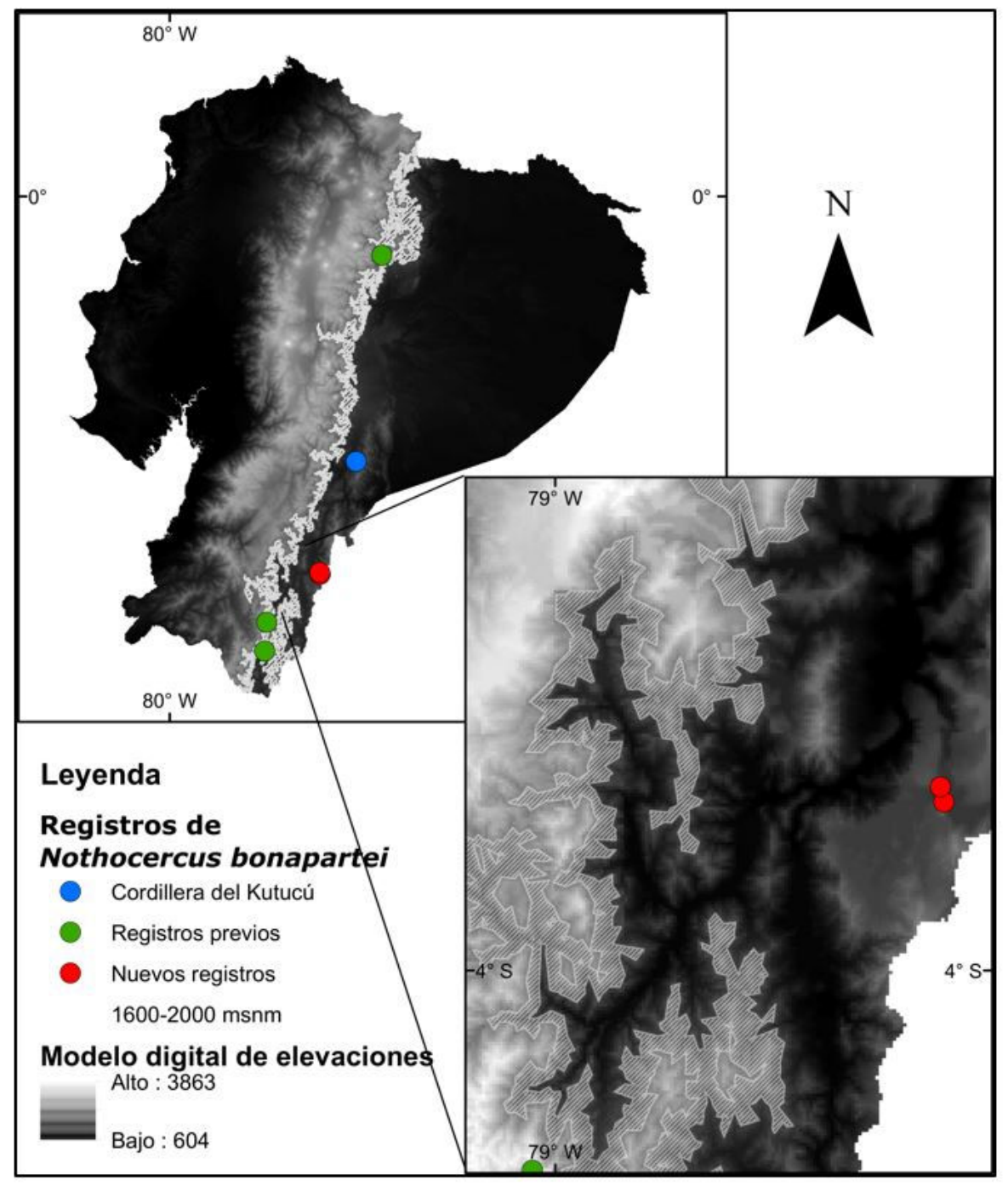

Figura 2: Mapa de distribución de Nothocercus bonapartei en Ecuador tomado de Ridgely \& Greenfield (2006) (área sombreada). Los puntos verdes corresponden a registros previos documentados de la especie en los Andes de Ecuador (Cosanga - Napo; Estación Científica San Francisco, Reserva Tapichalaca, cordillera de Numbala - Parque Nacional Podocarpus, Cerro Romerillos, La Canela) (MECN, eBird, J. Freile com. pers.), el punto azul se ubica en la cordillera de Kutukú (Robins et al., 1987); y, los puntos rojos corresponden a los registros en la cordillera del Cóndor presentados en este documento.

Es fundamental que este tipo de información sea utilizada en la toma de decisiones locales para fortalecer los procesos de planificación del uso del territorio de esta singular y frágil región, solo así se podrá asegurar la permanencia a largo plazo de la biodiversidad asociada a la misma y de los servicios ambientales que provienen de ella.

\section{AGRADECIMIENTOS}

Este trabajo se desarrolló con permiso de investigación del Ministerio del Ambiente No 15-2012-IC-FAU-DPAP-MA. Agradecemos a la Compañía Aurelian Ecuador S.A. - Kinross (actualmente Lundin Gold), quienes financiaron la ejecución de la campaña de campo para evaluaciones faunísticas en el Alto Machinaza. La fase de campo contó con la valiosa colaboración de los guías locales: Manuel Jima y Luis Gualán. Al Museo Ecuatoriano de Ciencias Naturales (MECN) por compartirnos los datos de especímenes colectados de la especie en Ecuador. Un agradecimiento especial a Boris Tinoco (Universidad del Azuay) por sus valiosas observaciones y aportes a las versiones iniciales de este documento; y, a los dos revisores anónimos cuyos comentarios permitieron consolidar el mismo. 


\section{REFERENCIAS}

Ágreda, A., Nilsson, J., Tonato, L., \& Román, H. (2005). A new population of Cinnamon-breasted Tody-Tyrant ((Hemitriccus cinnamomeipectus) in Ecuador. Cotinga, 24, 16-18.

Balchin, C.S., \& Toyne, E.P. (1998). The avifauna and conservation status of the Río Nangaritza Valley, Southern Ecuador. Bird Conservation International, 8, 237-253. doi: http://dx.doi.org/10.1017/S0959270900001908

BirdLife International. (2015). Nothocercus bonapartei. The IUCN Red List of Threatened Species 2012: e.T22678154A40069601. Recuperado de: http://dx.doi.org/10.2305/IUCN.UK.2012-1.RLTS.T22678154A40069601.en.

Brennan, P. (2009). Incubation in Great Tinamou (Tinamus major). The Wilson Journal of Ornithology 121(3), 506-511 doi: http:// dx.doi.org/10.1676/08-073.1

Cabot, J., Jutglar, F., Sharpe, C.J., \& Kirwan, G.M. (2001). Highland Tinamou (Nothocercus bonapartei). En: J. del Hoyo, A. Elliott, J. Sargatal, D.A. Christie, E. \& de Juana, (Eds), Handbook of the Birds of the World Alive. Barcelona: Lynx Editions. Recuperado de: http://www.hbw.com/node/52414

Fjeldså, J., \& Krabbe, N. (1990). Birds of the high Andes. Copenhagen: Zoological Museum.

Freile, J.F. (2009). Aves del Ecuador. En Boada, C., Freile, J. F., Jiménez, P., Nogales-Sornoza, F., \& Valencia, J. H. Fauna de Vertebrados del Ecuador (pp. 177-259). Loja: Universidad Técnica Particular de Loja.

Freile, J.F., \& Santander, T. (2005). Áreas Importantes para la Conservación de las Aves en Ecuador. Quito: Aves y Conservación, BirdLife International, Conservación Internacional y Ministerio del Ambiente de Ecuador.

Freile, J.F., Piedrahita, P., Buitrón-Jurado, G., Rodríguez, C.A., \& Bonacorso, E. (2011a). Aves de los Tepuyes de Nangaritza, Cordillera del Cóndor. En J. M. Guayasamin, \& E. Bonaccorso (Eds.). Evaluación Ecológica Rápida de la Biodiversidad de los Tepuyes de la Cuenca Alta del Río Nangaritza, Cordillera del Cóndor, Ecuador (pp 63-76). Quito: Conservación Internacional.

Freile, J.F., Piedrahita, P., Buitrón-Jurado, G., Rodríguez, C.A., Jadan, O., \& Bonacorso, E. (2011b). Observations on the natural history of the Royal Sunangel (Heliangelus regalis) in the Nangaritza Valley, Ecuador. The Wilson Journal of Ornithology 123(1), 85-92. doi: http://dx.doi.org/10.1676/10-054.1

Freile, J.F., Carrión, J.M., Prieto-Albuja, F., Suárez, L., \& Ortiz-Crespo, F. (2006). La ornitología en Ecuador: Un análisis del estado actual del conocimiento y sugerencias para prioridades de investigación. Ornitología Neotropical. 17, 183-202. Recuperado de: https://sora.unm.edu/sites/default/files/journals/on/v017n02/p0183-p0202.pdf

Freile, J.F., Krabbe, N., Piedrahita, P., Buitron-Jurado, G., Rodríguez-Saltos, C.A., Ahlman, F., Brinkhuizen D.M., \& Bonaccorso, E. (2014). Birds, Nangaritza River Valley, Zamora Chinchipe Province, southeast Ecuador: Update and revision. Check List 10(1), 54-71. doi: http://dx.doi.org/10.15560/10.1.54

Gomes, V. \& Kirwan, G.M. (2015). Highland Tinamou (Nothocercus bonapartei). En T.S. Schulenberg (Ed) Neotropical Birds Online. Ithaca: Cornell Lab of Ornithology. Recuperado de: http://neotropical.birds.cornell.edu/portal/species/overview?p_p_spp=56436

Handford, P., \& Mares, M.A. (1985). The mating systems of ratites and tinamous: an evolutionary perspective. Biological Journal of the Linnean Society, 25(1), 77-104. doi: http://dx.doi.org/10.1111/j.1095-8312.1985.tb00387.x

Hilty, S.L., \& Brown, W.L. (1986). A guide to the birds of Colombia. New Jersey: Princeton University Press.

Jadan, O., \& Aguirre, Z. (2011). Flora de los Tepuyes de la Cuenca Alta del Río Nangaritza, Cordillera del Cóndor. En: J.M. Guayasamin, \& E. Bonacorso (Eds.), Evaluación Ecológica Rápida de la biodiversidad de los Tepuyes de la Cuenca Alta del Río Nangaritza, Cordillera del Cóndor, Ecuador. Quito: Conservación Internacional.

Krabbe, N. \& Ahlman, L. (2009). Royal Sunangel (Heliangelus regalis) at Yankuam Lodge, Ecuador. Cotinga, 31, 132.

Krabbe, N., \& Nilsson, J. (2003). Birds of Ecuador: 1.24. (2003-2012). Sounds and photographs DVD ROM. Netherlands: Bird Songs International.

Krabbe, N., \& Sornoza, F. (1994). Avifaunistic results of a subtropical camp in the Cordillera del Condor, southeastern Ecuador. Bulletin of The British Ornithologists Club,114, 55-61.

Krabbe, N., Poulsen, B.O., Frolander, A., \& Rodríguez B., O. (1997). Range extensions of cloud forest birds from the high Andes of Ecuador: New sites for rare or little-recorded species. Bulletin British Ornithologists' Club, 117, 248-256. 
Loaiza, J.M., Sornoza, A.F., Agreda, A.E., Aguirre, J., Ramos, R., \& Canaday, C. (2005). The Presence of Wavy-breasted Parakeet Pyrrhura peruviana confirmed for Ecuador. Cotinga, 23. 37-38.

Lysinger, M., Moore, J.V., Krabbe, N., Coopmans, P., Lane, D., Navarrete, L., Nilsson, J., \& Ridgely, R. (2005). The Birds of Eastern Ecuador. Volume I: The Foothills and Lower Subtropics. USA: John V. Moore Nature Recordings.

McKay, W. D. (1980). Nest and young of the Highland Tinamou in southern Colombia. Condor, 82, 107. Recuperado de: https:// sora.unm.edu/sites/default/files/journals/condor/v082n01/p0107-p0108.pdf

McMullan, M., \& Navarrete, L. (2013). Fieldbook of the Birds of Ecuador, including the Galapagos Islands. Quito: Fundación de Conservación Jocotoco.

Miller, A. H. (1963). Seasonal activity and ecology of the avifauna of an American equatorial cloud forest. University of California Publications in Zoology, 66: 1-178.

Montalvo, L. D. (2012). Notas de distribución de aves en la Cordillera del Cóndor. Revista Politécnica 30(3), 172-178.

Moore, J. V., Krabbe, N., \& Jahn, O. (2013). Bird Sounds of Ecuador: A Comprehensive Collection. San José, California: John V. Moore Nature Recordings.

Neill, D. (2005). Cordillera del Cóndor. Botanical treasures between the Andes and the Amazon. Plant Talk, 41, 17-21.

Ordóñez-Delgado, L. (2012a). XC124860 Tinamú serrano Nothocercus bonapartei plumbeiceps. Available from http://www.xeno-canto. org/124860

Ordóñez-Delgado, L. (2012b). XC124266 Tinamú serrano Nothocercus bonapartei plumbeiceps. Available from http://www.xeno-canto. org/124266

Ordóñez-Delgado, L. (2012c). XC124265 Tinamú serrano Nothocercus bonapartei plumbeiceps. Available from http://www.xeno-canto. org/124265

Ordóñez-Delgado, L. (2012d). XC124264 Tinamú serrano Nothocercus bonapartei plumbeiceps. Available from http://www.xeno-canto. org/124264

Ordóñez-Delgado, L. (2012e). XC 124861 Tinamú serrano Nothocercus bonapartei plumbeiceps. Available from http://www. xeno-canto.org/124861

Ridgely, R.S., \& Greenfield, P.J. (2001). The birds of Ecuador. Ithaca: Cornell University Press.

Ridgely, R.S. \& Greenfield, P.J. (2006). Aves del Ecuador, guía de campo. Quito: Academy of Natural Science of Philadelphia y Fundación de Conservación Jocotoco.

Robbins, M.B., Ridgely, R.S., Schulenberg, T.S., \& Gill, F.B. 1987. The avifauna of the Cordillera de Cutucú, Ecuador, with comparisons to other Andean localities. Proceedings of the Academy of Natural Sciences of Philadelphia, 129(1), 243-259.

Schulenberg, T. S., \& Awbrey, K. (Eds.). (1997). The Cordillera del Condor Region of Ecuador and Peru: A Biological Assessment. RAP Working Papers 7. Washington, D.C: Rapid Assessment Program, Conservation International.

Schulenberg, T.S., Stotz, D. F., Lane, D.F., O'Neill, J.P., \& Parker, T.A. (2007). Birds of Peru. Princenton: Princenton University Press.

Sierra, R., Cerón, C., Palacios, W. \& Valencia, R. (1999). Propuesta preliminar de un Sistema de clasificación de vegetación para el Ecuador Continental. Quito: Proyecto INEFAN/GEF-BIRF y ECOCIENCIA.

Slud, P. (1964). The birds of Costa Rica. Distribution and ecology. Bulletin of the American Museum of Natural History, 128, 1-430. 